\title{
Nucleon spectral function from QCD sum rules with MEM
}

\author{
Keisuke Ohtani* \\ Tokyo Institute of Technology, Meguro, Tokyo 152-8551, Japan \\ E-mail: ohtani.keth.phys.titech.ac.jp
}

\section{Philipp Gubler}

RIKEN Nishina Center, Hirosawa 2-1, Wako, Saitama, 351-0198, Japan

E-mail: pgublereriken.jp

\section{Makoto Oka}

Tokyo Institute of Technology, Meguro, Tokyo 152-8551, Japan

J-PARC Branch, KEK Theory Center, Institute of Particle and Nuclear Studies, 203-1, Shirakata,

Tokai, Ibaraki, 319-1106, Japan

E-mail: oka@th.phys.titech.ac.jp

The nucleon and its negative-parity excited states in nuclear matter are examined in a maximum entropy method (MEM) analysis of QCD sum rules. We construct the parity projected nucleon QCD sum rules with a phase-rotated Gaussian kernel. This sum rule in vacuum has the interesting feature that it is dominated by the term of the chiral condensate. At the finite density, the positive parity OPE data is strongly modified while negative parity OPE data are hardly changed. This behavior is attributed to the fact that not only the chiral condensate term but also the $\left\langle q^{\dagger} q\right\rangle$ term has a large contribution in this sum rule. Analyzing this sum rule by MEM, we are able to study the modification of both the positive and negative parity states.

XV International Conference on Hadron Spectroscopy-Hadron 2013

4-8 November 2013

Nara, Japan

\footnotetext{
* Speaker.
} 


\section{Introduction}

The QCD sum rule method is a powerful tool for studying hadron properties directly from QCD [1]. In this method, the correlation function of an interpolating field operator coupled to the hadron of interest, which can be calculated in the deep Euclidean region by the operator product expansion (OPE), is related to the hadronic spectral function in the physical region by a dispersion relation. The non-perturbative contributions in the correlation function are expressed by vacuum condensates such as the chiral condensate $\langle\bar{q} q\rangle$. Since $\langle\bar{q} q\rangle$ is an order parameter of the chiral transition, we can investigate the relation between the hadron mass and chiral symmetry breaking.

In the traditional analysis, it is necessary to assume some specific functional form for the spectral function such as the "pole + continuum"-ansatz, where the pole corresponds to the lowest lying state of the hadron of interest and the continuum stands for contribution of other states. Although it is not completely obvious that the actual form of spectral function is similar to "pole + continuum"-ansatz, the properties of many hadrons have been successfully investigated. On the other hand, our approach with the help of the Maximum Entropy Method (MEM) is able to extract the spectral functions without any assuming specific form [2, 3, 4, 5, 6]. This feature is suitable for the analyses at finite density because the spectral function will be more complicated. We have applied this analysis method of QCD sum rules to the nucleon and its negative parity excited state in nuclear matter.

\section{Nucleon QCD sum rules}

In QCD sum rules of the nucleon channel in the nuclear matter, one usually studies the properties of the time ordered correlation function:

$$
\Pi(q)=i \int d^{4} x e^{i q x}\left\langle\Psi_{0}|T[\eta(x) \bar{\eta}(0)]| \Psi_{0}\right\rangle=\not \Pi_{1}(q)+\Pi_{2}(q)+\not \Pi_{u}(q)
$$

Here, $\eta, u$ and $\Psi_{0}$ are a nucleon interpolating field, the velocity of nuclear matter and the ground state of nuclear matter, respectively. Note that the nucleon interpolating field couples to both positive and negative parity states [7]. Therefore, when only the sum rule constructed from each componert $\Pi_{1}(q), \Pi_{2}(q)$ or $\Pi_{u}(q)$, the analysis is strongly disturbed by contributions of the opposite parity states. To remedy this problem, we use the old-fashioned correlator [8]:

$$
\Pi^{\text {old }}(q)=i \int d^{4} x e^{i q x} \theta\left(x_{0}\right)\left\langle\Psi_{0}|T[\eta(x) \bar{\eta}(0)]| \Psi_{0}\right\rangle,
$$

where the essential difference to Eq. 2.10 is the insertion of the Heaviside step-function $\theta\left(x_{0}\right)$ before carrying out the Fourier transform. Using the property that the old-fashioned correlator is analytic in the upper half of the complex $q_{0}$ plane, we get the parity projected sum rule:

$$
\int_{-\infty}^{\infty} d q_{0} \frac{1}{\pi} \operatorname{Im}\left[\Pi_{\mathrm{OPE}}^{ \pm}(q)\right] W(q)=\int_{0}^{\infty} d q_{0} \rho_{\mathrm{Phys} .}^{ \pm}(q) W(q) .
$$

Here, $\Pi_{\mathrm{OPE}}^{ \pm}(q)$ is calculated by the OPE in the deep Euclidean region, $\rho_{\mathrm{Phys}}^{ \pm}(q)$ stands for the physical spectral function of positive and negative parity states and $W(q)$ is an arbitrary analytic function in the upper half of the imaginary plane and real on the real axis. To construct the final 

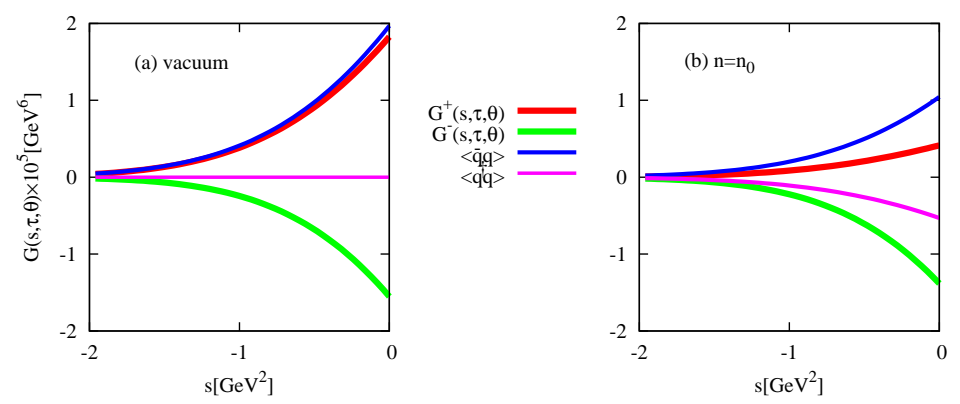

Figure 1: (a) $G_{\mathrm{OPE}}^{\mathrm{old} \pm}(s, \tau, \theta)$, chiral condensate $\langle\bar{q} q\rangle$ and $\left\langle q^{\dagger} q\right\rangle$ terms at $\tau=0.5\left[\mathrm{GeV}^{4}\right]$ and $\theta=0.108 \pi$ in the vacuum. The thick lines denote $G_{\mathrm{OPE}}^{\text {old }}(s, \tau, \theta)$ and the thin lines are $\langle\bar{q} q\rangle$ and $\left\langle q^{\dagger} q\right\rangle$ terms. (b) Same as for (a), but at the normal nuclear matter density $n_{0}$.

sum rule, we have to specify the kernel $W(q)$. One usually use a Borel kernel: $W(q)=\exp \left(-\frac{q^{2}}{\mathrm{M}^{2}}\right)$ or Gaussian kernel: $W(q)=\frac{1}{\sqrt{4 \pi \tau}} \exp \left(-\frac{\left(q^{2}-s\right)^{2}}{4 \tau}\right)$, which correspond to the Borel and Gaussian sum rules, respectively. However, in these sum rules, the $\alpha_{s}$ corrections for the perturbative and four quark condensate terms and contributions of the continuum are large, which lower the reliability of the analyses [9]. Following the method proposed by Ioffe and Zyablyuk [10], who have constructed a new class of sum rules by using the phase rotated complex variable $q^{2} e^{i \theta}$ instead of the real $q^{2}$, we were able to improve this situation. One advantage of this approach lies in the possibility of suppressing certain terms of the OPE by choosing some specific value of $\theta$. To apply this approach to the parity projected sum rules, we use the phase-rotated kernel:

$$
W(q, s, \tau, \theta) d q_{0}=\frac{1}{\sqrt{4 \pi \tau}} \operatorname{Re}\left[\left(q_{0}-|\vec{q}|\right) e^{-i \theta} \exp \left(-\frac{\left(q^{2} e^{-2 i \theta}-s\right)^{2}}{4 \tau}\right) e^{-i \theta} d q_{0}\right] .
$$

We can obtain the specific form of the left hand side of Eq. 2.3) which is defined as $G_{\mathrm{OPE}}^{\mathrm{old} \pm}(s, \tau, \theta)$ :

$$
\begin{aligned}
G_{\mathrm{OPE}}^{\mathrm{old} \pm}(s, \tau, \theta)= & \left(C_{0}(\theta)+C_{0 \alpha_{s}}(\theta)\right)+\left(C_{3}^{\prime}(\theta)+C_{3 \alpha_{s}}^{\prime}(\theta)\right)\left\langle q^{\dagger} q\right\rangle+C_{4}(\theta)\left\langle\frac{\alpha_{s}}{\pi} G^{2}\right\rangle+\cdots \\
& \pm\left[\left(C_{3}(\theta)+C_{3 \alpha_{s}}(\theta)\right)\langle\bar{q} q\rangle+C_{5}(\theta)\langle\bar{q} g \sigma \cdot G q\rangle+\cdots\right]
\end{aligned}
$$

where $C_{n}$ are numerical coefficients and $n$ stands for the dimension of the condensates and \langle\rangle represents the expectation value with respect to the ground state of nuclear matter. As for the density dependence of the condensates, we use the linear density approximation, which is accepted as valid up to the normal nuclear matter density. Using the phase rotation, the ratios of $\alpha_{s}$ corrections to leading terms at dimension $0: \frac{C_{0 \alpha_{s}}}{C_{0}}$ is reduced from $90 \%$ to $5 \%$ at $\theta=0.108 \pi$ taking $\alpha_{s}=0.5$, which shows that the convergence of the perturbative expansion is significantly improved. The chiral condensate term, $\left\langle q^{\dagger} q\right\rangle$ term and $G_{\mathrm{OPE}}^{\mathrm{old} \pm}(s, \tau, \theta)$ in the vacuum and at finite density are given in Fig. 1. It can be seen that in vacuum, the difference of the OPE data between the positive parity and negative parity states is caused by the chiral condensate. We also find that at finite density, the values of the positive parity OPE become small due to the decrease of the chiral condensate and 

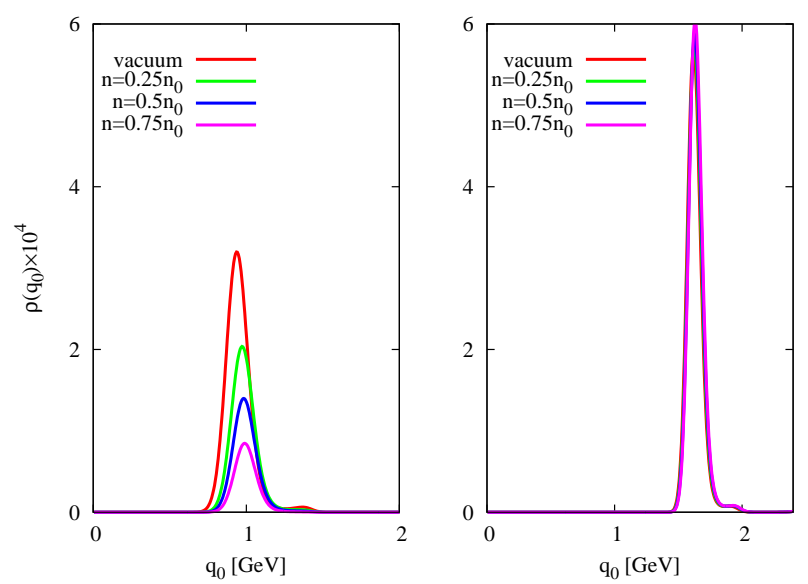

Figure 2: The density dependence of the positive (left) and negative parity (right) spectral functions extracted from MEM analyses of the OPE data $G_{\mathrm{OPE}}^{\mathrm{old}} \pm(s, \tau, \theta) . n_{0}$ stand for the normal nuclear matter density.

\begin{tabular}{|c|c|c|c|c|}
\hline density & vacuum & $0.25 n_{0}$ & $0.5 n_{0}$ & $0.75 n_{0}$ \\
\hline$M_{+}^{*}[\mathrm{MeV}]$ & 910 & 850 & 760 & 620 \\
\hline$\Sigma_{+}^{v}[\mathrm{MeV}]$ & 0 & 100 & 200 & 350 \\
\hline$M_{-}^{*}[\mathrm{MeV}]$ & 1580 & 1580 & 1600 & 1620 \\
\hline$\Sigma_{-}^{v}[\mathrm{MeV}]$ & 0 & 0 & 10 & 30 \\
\hline
\end{tabular}

Table 1: The density dependence of the positive and negative parity effective masses and self energies. Here $n_{0}$ represents the normal nuclear matter density. The parity of the corresponding states is shown on the subscript.

the increase of the $\left\langle q^{\dagger} q\right\rangle$. On the other hand, the values of the negative parity OPE at finite density are hardly changed because the in-medium modification of the condensates cancel each other.

\section{Results}

Carrying out the analysis using the OPE data $G_{\mathrm{OPE}}^{\mathrm{old} \pm}(s, \tau, \theta)$ with MEM, we obtain the corresponding spectral functions. The results are shown in Fig. 2 For the positive and negative parity spectral function in the vacuum, the peaks are found at $910 \mathrm{MeV}$ and $1580 \mathrm{MeV}$, respectively. At the finite density, the peak positions are hardly changed, which indicate that the energy of the both states are almost density independent. To investigate the effective masses and self energy, we consider a specific form of the nucleon propagator. Using the mean field approximation, the propagator $G(q)$ can be described as

$$
G(q)=\frac{\phi^{*}+M^{*}}{\left(q^{*}\right)^{2}-\left(M^{*}\right)^{2}+i \varepsilon} .
$$

Here, $q_{\mu}^{*}=q_{\mu}-u_{\mu} \Sigma_{v}$ and the effective mass $M^{*}$ is defined as $M+\Sigma_{s}$. Fitting the components $\Pi_{1}$, $\Pi_{2}$ and $\Pi_{u}$, we derive the effective masses and the vector self energies. The results are shown in 
Table 1. For the positive parity state, as the density increase, the effective mass decreases while the vector self energy increases. On the other hand, the effective mass and the vector self energy of the negative parity state are scarcely changed. This behavior is a consequence of the density dependence of the OPE data.

\section{Summary}

We have constructed parity projected nucleon QCD sum rule in the vacuum and at finite density taking into account the first order $\alpha_{s}$ corrections. Furthermore using the phase-rotated kernel, we improve some technical problems of the usual nucleonic sum rules. This sum rule has the interesting feature that in vacuum, the splitting between positive and negative parity states are caused by the spontaneous symmetry breaking of the chiral symmetry since the term of the chiral condensate which switches its sign depending on the parity is dominant in the OPE data. We also find that the values of the positive parity OPE strongly depend on the density but its values of the negative parity states are almost density independent.

We have analyzed the nucleon spectral function using the derived sum rules and the maximum entropy method and extracted information of both the positive and negative parity states. For the positive parity states at the finite density, we find that the effective mass decreases and the vector self energy increases. For the negative parity states at the finite density, the effective mass and the vector self energy are hardly changed. The detailed discussion of the relation between the behavior of the OPE data and the spectral function will be given in a forthcoming publication [11].

\section{References}

[1] M.A. Shifman, A.I. Vainshtein, and V.I. Zakharov, Nucl. Phys. B147, 385 (1979).

[2] P. Gubler and M. Oka, Prog. Theor. Phys. 124, 995 (2010).

[3] K. Ohtani, P. Gubler and M. Oka, Eur. Phys. J. A 47, 114 (2011).

[4] P. Gubler, K. Morita and M. Oka, Phys. Rev. Lett. 107, 092003 (2011).

[5] K. Suzuki, P. Gubler, K. Morita and M. Oka, Nucl. Phys. A897 28 (2013).

[6] K. Ohtani, P. Gubler and M. Oka, Phys. Rev. D 87, 034027 (2013).

[7] Y. Chung, H.G. Dosch, M. Kremer and D. Schall, Nucl. Phys. B197, 55 (1982).

[8] D. Jido, N. Kodama and M. Oka, Phys. Rev. D 54, 4532 (1996).

[9] D.B. Leinweber, Ann. Phys. (N.Y.) 254, 328 (1997).

[10] B. L. Ioffe and K.N. Zyablyuk, Nucl. Phys. A687, 437 (2001).

[11] K. Ohtani, P. Gubler and M. Oka, preparation. 